This item was submitted to Loughborough's Research Repository by the author.

Items in Figshare are protected by copyright, with all rights reserved, unless otherwise indicated.

\title{
A nonlinear disturbance observer for robotic manipulators
}

PLEASE CITE THE PUBLISHED VERSION

PUBLISHER

(C) IEEE

LICENCE

CC BY-NC-ND 4.0

\section{REPOSITORY RECORD}

Chen, Wen-Hua, Donald J. Ballance, Peter J. Gawthrop, and John O'Reilly. 2008. "A Nonlinear Disturbance Observer for Robotic Manipulators". figshare. https://hdl.handle.net/2134/3759. 
This item was submitted to Loughborough's Institutional Repository (https://dspace.lboro.ac.uk/) by the author and is made available under the following Creative Commons Licence conditions.

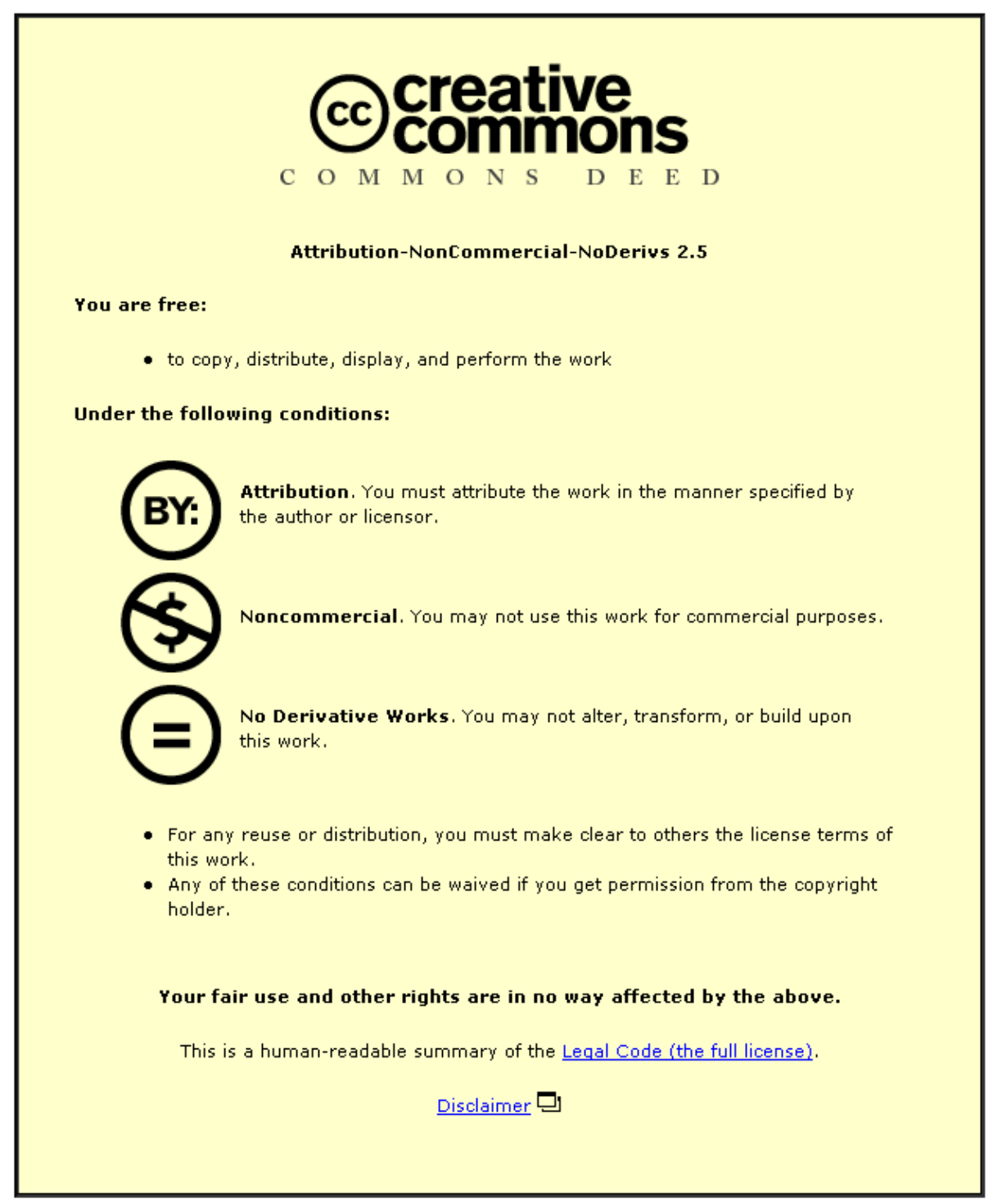

For the full text of this licence, please go to: http://creativecommons.org/licenses/by-nc-nd/2.5/ 


\title{
A Nonlinear Disturbance Observer for Robotic Manipulators
}

\author{
Wen-Hua Chen, Member, IEEE, Donald J. Ballance, Member, IEEE, Peter J. Gawthrop, Senior Member, IEEE, \\ and John O'Reilly, Senior Member, IEEE
}

\begin{abstract}
A new nonlinear disturbance observer (NDO) for robotic manipulators is derived in this paper. The global exponential stability of the proposed disturbance observer (DO) is guaranteed by selecting design parameters, which depend on the maximum velocity and physical parameters of robotic manipulators. This new observer overcomes the disadvantages of existing DO's, which are designed or analyzed by linear system techniques. It can be applied in robotic manipulators for various purposes such as friction compensation, independent joint control, sensorless torque control, and fault diagnosis. The performance of the proposed observer is demonstrated by the friction estimation and compensation for a two-link robotic manipulator. Both simulation and experimental results show the NDO works well.
\end{abstract}

Index Terms-Friction, nonlinear estimation, observers, robots.

\section{INTRODUCTION}

D ISTURBANCE observers (DO's) have been used in robotic manipulator control for a long time. In general, the main objective of the use of DO's is to deduce external unknown or uncertain disturbance torques without the use of an additional sensor. The use of DO's in robotic manipulators can be divided into the following categories.

1) Independent joint control is widely used in industrial robots where a multilink manipulator is divided into several independent links with linear dynamics. The performance of these kind of controllers can be improved by the use of a DO. This is accomplished by considering the coupling torques from other links as an unknown external disturbance and using a DO to estimate and compensate for it [1]. This technique has also been extended to deal with parameter variations and unmodeled dynamics whereby it improves the robustness of robots [2];

2) Friction is a common phenomenon in mechanical systems and plays an important role in system performance. Many friction models and compensation methods have been proposed [3]. One of the most promising methods

\footnotetext{
Manuscript received December 22, 1998; revised March 23, 2000. Abstract published on the Internet April 21, 2000. An earlier version of this paper was presented at the 38th IEEE Control and Decision Conference, Phoenix, AZ, December 7-10, 1999. This work was supported by the U.K. Engineering and Physical Sciences Research Council under Grant GR/L 62665.

W.-H. Chen and J. O'Reilly are with the Centre for Systems and Control, Department of Electronics and Electrical Engineering, University of Glasgow, Glasgow G12 8QQ, U.K. (e-mail: w.chen@elec.gla.ac.uk).

D. J. Ballance and P. J. Gawthrop are with the Centre for Systems and Control, Department of Mechanical Engineering, University of Glasgow, Glasgow G12 8QQ, U.K. (e-mail: D.Ballance@mech.gla.ac.uk).

Publisher Item Identifier S 0278-0046(00)06805-2.
}

is observer-based control where a DO is used to estimate friction [4];

3) DO's have been used in robotic manipulators for force feedback and hybrid position/force control where the DO works as a torque sensor [5]-[7]. In this case, it is supposed that the friction and other dynamics are well modeled and compensated for. The use of a DO, rather than several torque sensors (for each link, at least one torque sensor is required), simplifies the structure of the system, reduces the cost, and improves the reliability. With this technique, sensorless torque control can be implemented [5]-[7].

4) Robotic manipulators work in a dynamic highly uncertain environment. In this application, DO's provide signals for monitoring and trajectory planning rather than for control. For example, in robotic assembly when the component is misinserted, the reaction torque/force is greatly increased and could damage the robotic manipulator. A DO can estimate the reaction torque online and transmit this information to the monitoring or planning level. Chan [8] uses a DO in electronic component assembly, while Ohishi and Ohde [9] give an example of the use of a DO in collision. Although the DO technique has been widely used in robotic manipulator control for various purposes, in almost all cases, the analysis or design is based on linearized models or using linear system techniques. Since a multilink robotic manipulator is a highly nonlinear and coupled system, the validity of using linear analysis and synthesis techniques may be doubtful. Many important properties of existing DO's have not been established, e.g., unbiased estimation or even global stability. There are, however, some recent results using nonlinear disturbance observers (NDO's). A variable structure DO has been proposed [10] and a nonlinear observer for a special kind of friction, i.e., Coulomb friction, has been proposed by Friedland and Park [11]. This nonlinear observer is designed based on the model of Coulomb friction, and the global convergence ability has been shown. It has been further modified and implemented on robotic manipulators by Tafazoli et al. [12]. However, a specific model of friction will not be used in this paper, and the whole design is based on the DO concept. That is, similar to other unknown torques, the friction is considered as a disturbance on the control torque.

It should be stressed that the DO rather than a velocity observer of a manipulator is considered in this paper. A velocity observer has been considered by many authors. Bona and Indri have compared and implemented several linear and nonlinear velocity observers on a robotic manipulator [13]. 
An NDO for multilink robotic manipulators will be presented in this paper. By carefully selecting the observer gain function, it will be shown that global convergence is guaranteed. This result is based on Lyapunov theory.

\section{PRoblem Formulation AND A BASIC OBSERVER}

\section{A. Problem Formulation}

For the sake of simplicity, a two-link robotic manipulator is considered in this paper. The main idea is readily extended to the more general case. The model of a two-link robotic manipulator can be represented by

$$
J(\theta) \ddot{\theta}+G(\theta, \dot{\theta})=T+d
$$

where $\theta \in R^{2}, \dot{\theta} \in R^{2}$, and $T(t) \in R^{2}$ are displacement, velocity, and control vectors, respectively. When only the dynamics of the links are considered in the model, the control input $T$ is either the torque or force. $d \in R^{2}$ is a disturbance torque or force vector. It should be noted that $d$ has different meanings in different observer applications. For example, it can be friction in friction compensation, reaction torque or force in force control, and unmodeled dynamics in independent joint control. Since a general observer will be derived in this paper, all of them are considered as "disturbances." When the first-order dynamics of dc motors are included in the above model, $T$ is the voltage vector imposed on the motors instead of the torque vector. As a result, the torque disturbance is also equivalent to the disturbance on the voltage on the motors. Hence, $d$ is the disturbance voltage in this case.

The objective of this paper is to design an observer such that the estimation $\hat{d}$ yielded by the observer exponentially approaches the disturbance $d$ under any $\theta(t), \dot{\theta}(t)$, and $t \in\left[t_{0}, \infty\right)$.

\section{B. Initial Observer}

A basic idea in the design of observers/estimators is to modify the estimation by the difference between the estimated output and the actual output. Since (1) can be written as

$$
d=J(\theta) \ddot{\theta}+G(\theta, \dot{\theta})-T
$$

a DO is proposed as

$$
\dot{\hat{d}}=-L(\theta, \dot{\theta}) \hat{d}+L(\theta, \dot{\theta})(J(\theta) \ddot{\theta}+G(\theta, \dot{\theta})-T) .
$$

Since, in general, there is no prior information about the derivative of the disturbance $d$, it is reasonable to suppose that

$$
\dot{d}=0
$$

which implies that the disturbance varies slowly relative to the observer dynamics. However, it will be illustrated by simulation and experiment that the observer developed in this paper can also track some fast time-varying disturbances.

Define the observer error

$$
e(t)=d-\hat{d}
$$

Combining (4) with the observer (3) yields

$$
\dot{e}=\dot{d}-\dot{\hat{d}}=L(\theta, \dot{\theta}) \hat{d}-L(\theta, \dot{\theta}) d
$$

That is, the observer error is governed by

$$
\dot{e}+L(\theta, \dot{\theta}) e=0 \text {. }
$$

It can be shown that the observer is globally asymptotically stable by choosing

$$
L(\theta, \dot{\theta})=\operatorname{diag}\{c, c\}
$$

where $c>0$. More specifically, the exponential convergence rate can be specified by choosing $c$.

\section{NDO}

The acceleration signal $\ddot{\theta}$ is not available in many robotic manipulators, and it is also difficult to construct the acceleration signal from the velocity signal by differentiation due to measurement noise. However, although the observer (3) is not practical to implement, it provides a basis for the further nonlinear observer design.

\section{A. Modified Observer}

Define an auxiliary variable vector

$$
z=\hat{d}-p(\theta, \dot{\theta})
$$

where $z \in R^{2}$. The designed function vector $p(\theta, \dot{\theta})$ is to be determined.

Let the function $L(\theta, \dot{\theta})$ in (3) be given by the following nonlinear equation:

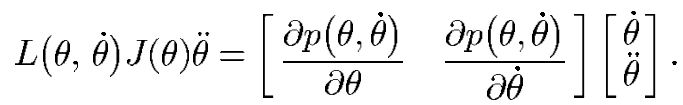

Invoking (9) and (10) with (3) yields

$$
\begin{aligned}
& \dot{z}=\dot{\hat{d}}-\frac{d p(\theta, \dot{\theta})}{d t} \\
& =\dot{\hat{d}}-\left[\begin{array}{ll}
\frac{\partial p(\theta, \dot{\theta})}{\partial \theta} & \frac{\partial p(\theta, \dot{\theta})}{\partial \dot{\theta}}
\end{array}\right]\left[\begin{array}{l}
\dot{\theta} \\
\ddot{\theta}
\end{array}\right] \\
& =-L(\theta, \dot{\theta})(z+p(\theta, \dot{\theta}))+L(\theta, \dot{\theta})(G(\theta, \dot{\theta})-T) \text {. }
\end{aligned}
$$

Hence, the NDO is given by

$$
\dot{z}=-L(\theta, \dot{\theta}) z+L(\theta, \dot{\theta})(G(\theta, \dot{\theta})-T-p(\theta, \dot{\theta}))
$$

and

$$
\hat{d}=z+p(\theta, \dot{\theta})
$$

where $L(\theta, \dot{\theta})$ is given by (10).

\section{B. Stability of the NDO}

It follows from (5), and (11)-(13) that the observer error equation is given by

$$
\dot{e}=\dot{d}-\dot{\hat{d}}=-\dot{z}-\frac{p(\theta, \dot{\theta})}{d t}=-L(\theta, \dot{\theta}) e .
$$


The estimation $\hat{d}$ approaches the disturbance $d$ if $L(\theta, \dot{\theta})$ is chosen such that (14) is asymptotically stable. Hence, the function $p(\theta, \dot{\theta})$ must be selected such that the function $L(\theta, \dot{\theta})$ given by (10) satisfies this condition. In general, it is not easy to select such a nonlinear function $p(\theta, \dot{\theta})$ especially for multivariable systems. However, for a multilink robotic manipulator, with the help of its characteristics, a systematic method for selecting the nonlinear function $p(\theta, \dot{\theta})$, such that the observer with $L(\theta, \dot{\theta})$ given by (10) is asymptotically stable, is developed in this paper.

The inertial matrix $J(\theta)$ for a two-link manipulator is given by [14]

$$
J(\theta)=\left[\begin{array}{cc}
j_{1}+2 X \cos \left(\theta_{2}\right) & j_{2}+X \cos \left(\theta_{2}\right) \\
j_{2}+X \cos \left(\theta_{2}\right) & j_{3}
\end{array}\right]
$$

where $j_{1}, j_{2}, j_{3}$, and $X$ are inertial parameters, which depend on the masses of the links, motors and tip load, and the lengths of the links.

Theorem: For the two-link robotic manipulator (1), when the function $p(\theta, \dot{\theta})$ in the observer (12) and (13) is chosen as

$$
p(\theta, \dot{\theta})=c\left[\begin{array}{c}
\dot{\theta}_{1} \\
\dot{\theta}_{1}+\dot{\theta}_{2}
\end{array}\right]
$$

and $c$ satisfies

$$
c>X \dot{\theta}_{2 m}
$$

where $\dot{\theta}_{2 m}$ denotes the maximum velocity of the second link, then the observer (12) and (13) is globally asymptotically stable.

Proof: Since $p(\theta, \dot{\theta})$ is given by (16), it yields

$$
\frac{d p(\theta, \dot{\theta})}{d t}=\left[\frac{\partial p(\theta, \dot{\theta})}{\partial \theta} \frac{\partial p(\theta, \dot{\theta})}{\partial \dot{\theta}}\right]\left[\begin{array}{l}
\dot{\theta} \\
\ddot{\theta}
\end{array}\right]=c\left[\begin{array}{ll}
1 & 0 \\
1 & 1
\end{array}\right] \ddot{\theta} .
$$

Combining the above equation with (10) gives

$$
L(\theta, \dot{\theta})=c\left[\begin{array}{ll}
1 & 0 \\
1 & 1
\end{array}\right] J(\theta)^{-1}
$$

since $J(\theta)$ is positive definite for all $\theta$ and $\dot{\theta}$ and, therefore, invertible.

From (15), $J(\theta)$ can be written as

$$
J(\theta)=\left[\begin{array}{ll}
1 & 1 \\
0 & 1
\end{array}\right] \bar{J}(\theta)\left[\begin{array}{ll}
1 & 0 \\
1 & 1
\end{array}\right]
$$

where

$$
\bar{J}(\theta)=\left[\begin{array}{cc}
j_{1}-2 j_{2}+j_{3} & j_{2}-j_{3}+X \cos \theta_{2} \\
j_{2}-j_{3}+X \cos \theta_{2} & j_{3}
\end{array}\right] .
$$

Hence,

$$
L(\theta)=c \bar{J}(\theta)^{-1}\left[\begin{array}{rr}
1 & -1 \\
0 & 1
\end{array}\right] .
$$

Since $\bar{J}(\theta)$ is also positive definite for all $\theta$, a Lyapunov function candidate for the observer (12) and (13) can be chosen as

$$
V(e, \theta)=e^{T \bar{J}}(\theta) e .
$$

Differentiating the Lyapunov function with respect to time $t$ along the observer trajectory gives

$$
\begin{aligned}
& \frac{d V(e, \theta)}{d t}= \frac{\partial V(e, \theta)}{\partial e} \dot{e}+\frac{\partial V(e, \theta)}{\partial \theta} \dot{\theta} \\
&=-c e^{T}\left[\begin{array}{rr}
1 & -1 \\
0 & 1
\end{array}\right] e-c e^{T}\left[\begin{array}{rr}
1 & 0 \\
-1 & 1
\end{array}\right] e \\
& \quad+e^{T}\left[\begin{array}{cc}
0 & -X \dot{\theta}_{2} \sin \theta_{2} \\
-X \dot{\theta}_{2} \sin \theta_{2} & 0
\end{array}\right] e \\
&=-e^{T}\left[\begin{array}{cc}
2 c & -c+X \dot{\theta}_{2} \sin \theta_{2} \\
-c+X \dot{\theta}_{2} \sin \theta_{2} & 2 c
\end{array}\right] e .
\end{aligned}
$$

Hence, $(d V(e, \theta) / d t)<0$ for all $e$ and $\theta, \dot{\theta}$ if

$$
\left|\begin{array}{cc}
2 c & -c+X \dot{\theta}_{2} \sin \theta_{2} \\
-c+X \dot{\theta}_{2} \sin \theta_{2} & 2 c
\end{array}\right|>0 .
$$

That is,

$$
\left(3 c-X \dot{\theta}_{2} \sin \theta_{2}\right)\left(c+X \dot{\theta}_{2} \sin \theta_{2}\right)>0 .
$$

Since $c$ satisfies (17), the above inequality is met. The equilibrium point $(e=0)$ is then globally asymptotically stable.

From the Theorem, the stability of the observer depends on the maximum velocity of the second link and other physical parameters. By choosing the design parameter $c$ satisfying the inequality (17), the global stability of the observer is guaranteed.

\section{Convergence Rate of the NDO}

It can be shown the minimum eigenvalue of the matrix in (25) for all $\theta$ is given by

$$
q_{1}=c-X \dot{\theta}_{2 m}
$$

Thus,

$$
\frac{d V(\theta, e)}{d t} \leq-q_{1}\|e\|^{2} .
$$

Let $q_{2}$ denote the maximum eigenvalue of the matrix $\hat{J}(\theta)$ for all $\theta$. It then follows from (24) that

$$
V(t) \leq V\left(t_{0}\right) e^{-\left(q_{1} / q_{2}\right)\left(t-t_{0}\right)} .
$$

The speed of convergence is bounded by $q_{1} / q_{2}$. For a robotic manipulator, $q_{2}$ and the prescribed maximum velocity are fixed. By choosing the parameter $c$, the desired exponential convergence rate can be achieved.

\section{Simulation AND EXPERIMENTAL RESUlts}

The proposed NDO is tested in this section. As stated in Section I, the NDO can be used in robotic manipulators for various purposes. In what follows, the NDO is designed as a friction observer. That is, the NDO is used to estimate the friction for a two-link robotic manipulator. The reason for designing a friction observer here is that friction varies rapidly, even discontinuously. It is a challenging task in observer design. The simulation and experiment are based on a two-link manipulator with 


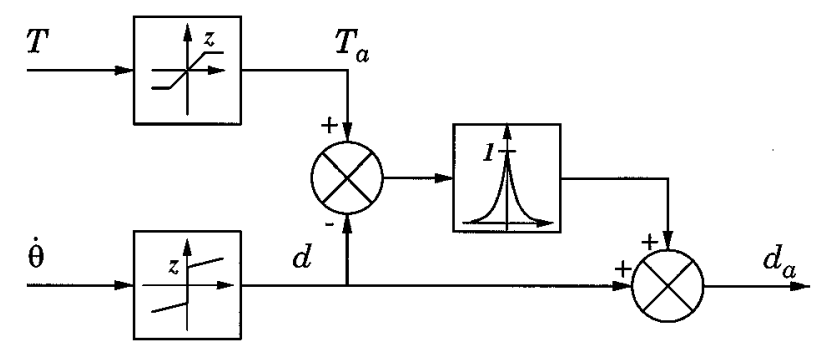

Fig. 1. Structure of the revised friction model.

$\mathrm{dc}$ motor actuators. The dynamic model of the manipulator including the first-order de motor dynamics is governed by (1) and is detailed in [15].

\section{A. Friction Simulation}

The friction considered is Coulomb and Viscous friction, given by

$$
d(\dot{\theta})=z \operatorname{sign}(\dot{\theta})+k \dot{\theta} .
$$

The parameters for first and second links in the simulation are given by

$$
\begin{aligned}
z & =0.0541 \mathrm{~N} \cdot \mathrm{m} \\
k & =0.00676 \mathrm{~N} \cdot \mathrm{m} / \mathrm{rad} / \mathrm{s}
\end{aligned}
$$

and

$$
\begin{aligned}
& z=0.0176 \mathrm{~N} \cdot \mathrm{m} \\
& k=0.0088 \mathrm{~N} \cdot \mathrm{m} / \mathrm{rad} / \mathrm{s}
\end{aligned}
$$

respectively.

There are some problems in using the friction model (30) in simulation directly. One is due to the discontinuity of the friction characteristics at zero velocity - a very small step size is required for testing zero velocity. The other is that when the velocity is zero, or the system is stationary, the friction is indefinite and depends on the controlled torque. In the simulation, to improve the numerical efficiency, a revised friction model, which is modified from [16], is adopted. The structure for the revised friction model is given by Fig. 1. It can be described by the following mathematical model:

$$
d_{a}=d+\left(T_{a}-d\right) e^{-(\dot{\theta} / l)^{2}}
$$

where $d$ is given by (30), $d_{a}$ is the revised friction and $l$ is a small positive scalar, and $T_{a}$ is given by

$$
T_{a}=\left\{\begin{array}{rlrl}
Z, & T & >Z \\
T, & -Z \leq T \leq Z \\
Z, & T<-Z .
\end{array}\right.
$$

When the velocity is within a very small area near zero, defined by $l$, the friction $d_{a}$ is equal to the applied torque $T$. When the velocity is greater than this, the second term in the above expression vanishes and the friction $d_{a}$ given by this revised model is equal to the friction given by (30). To compare the accuracy of the revised friction model (33), the friction defined by the classic Coulomb and viscous friction model (30) and the revised model

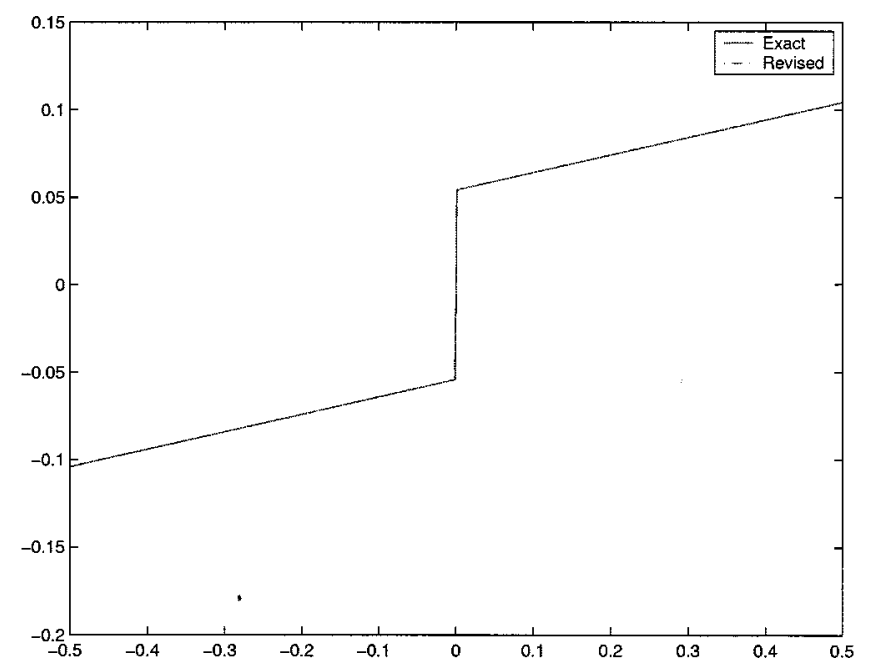

Fig. 2. Comparison of the Columb and Viscous friction model and the revised friction model.

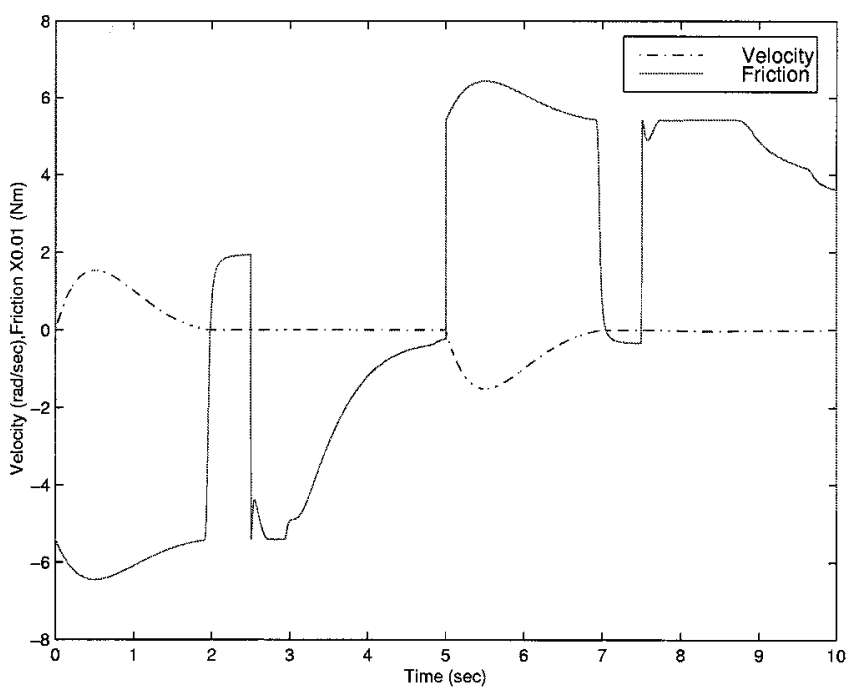

Fig. 3. First-link velocity and friction time histories.

(33) for first link is shown in Fig. 2. In this figure, $l$ is chosen as 0.001 . The frictions given by these two models are almost indistinguishable. However, experience has shown that using the revised model greatly improves the computational efficiency for simulation.

\section{B. Simulation Results}

A controller is designed by computed torque control where the disturbance is not taken into account. When square-wave command references are given for first and second links, respectively, the velocity and friction histories are shown in Figs. 3 and 4. It can be seen that the friction varies very rapidly with the velocity.

A friction observer is designed by the NDO technique developed in this paper. The design parameter $c$ is chosen as 50 . The estimation given by the observer (12) and (13) is shown by Figs. 5 and 6 . The observer exhibits excellent tracking performance. It successfully estimates the friction on-line and no friction model is required. 


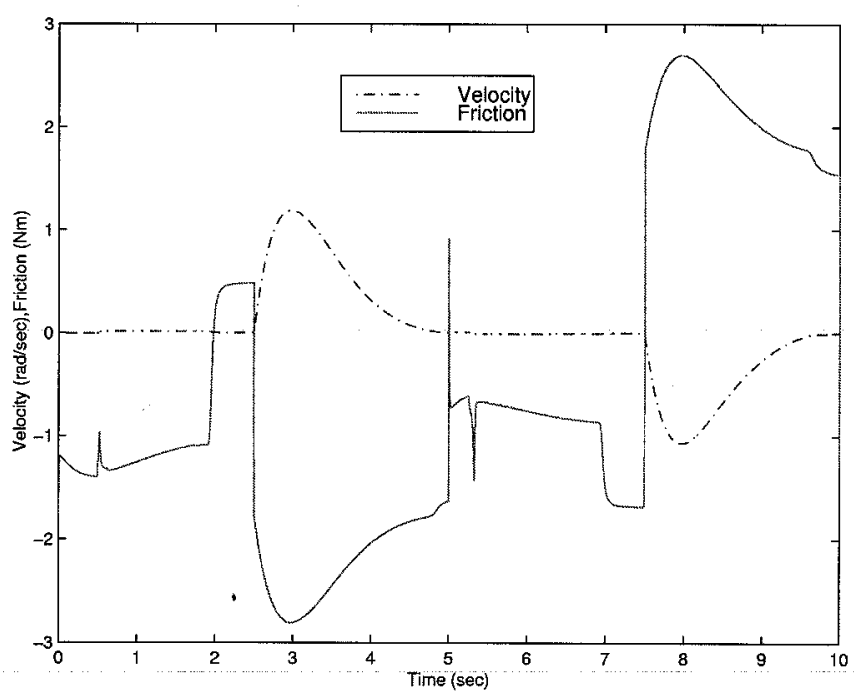

Fig. 4. Second link velocity and friction time histories.

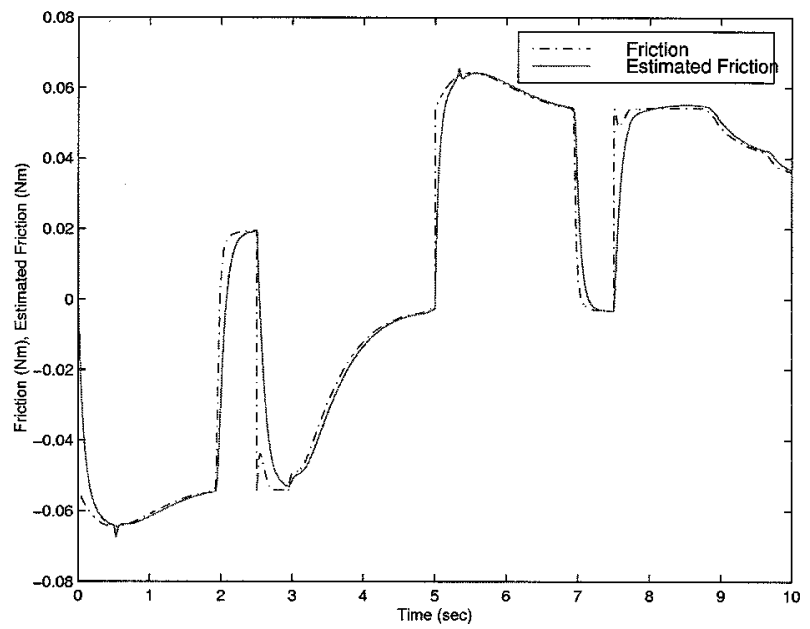

Fig. 5. First-link friction estimated by the friction observer.

\section{Experimental Results}

The proposed NDO is implemented on a two-link robotic manipulator in the laboratory. The experimental results for a computed torque controller with and without the NDO are compared. The control structure, which combines a computed torque controller with the NDO, is shown in Fig. 7, where the effect of the friction is compensated for by the outputs of the NDO via feedforward. With the NDO, the parameter $c$ is chosen as one.

Figs. 8-11 show the results of computed torque control with and without the NDO, in each case showing both the first and second links. Fig. 8 shows the reference signals (dotted-dashed) and the position tracking performance for both the computed torque control without the NDO (dashed) and with the NDO (solid line).

The friction estimated by the NDO is plotted in Fig. 9. When the friction of the robot is compensated by outputs of the NDO, under the same controller and reference signal as used for the case without the NDO, the tracking performance is shown in

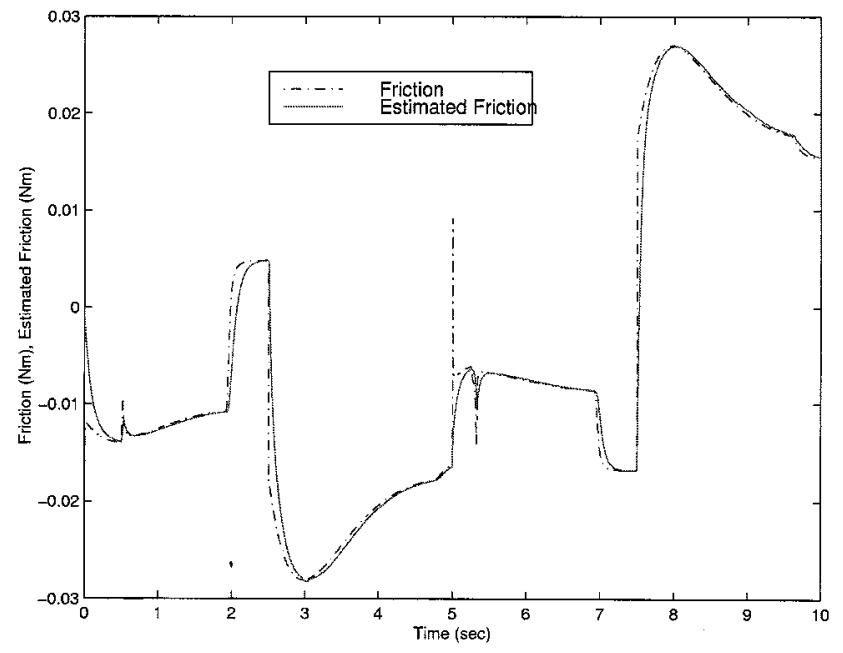

Fig. 6. Second-link friction estimated by the friction observer.

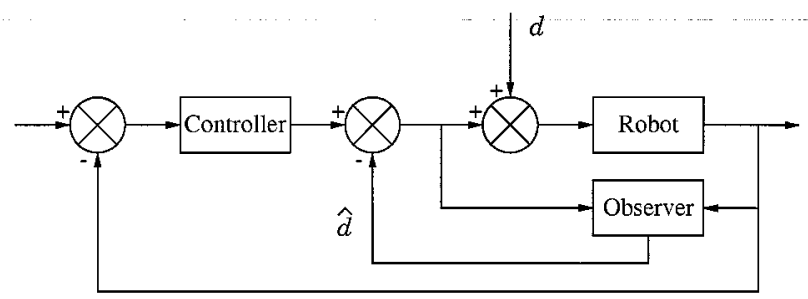

Fig. 7. Robotic manipulator controller structure with the NDO.
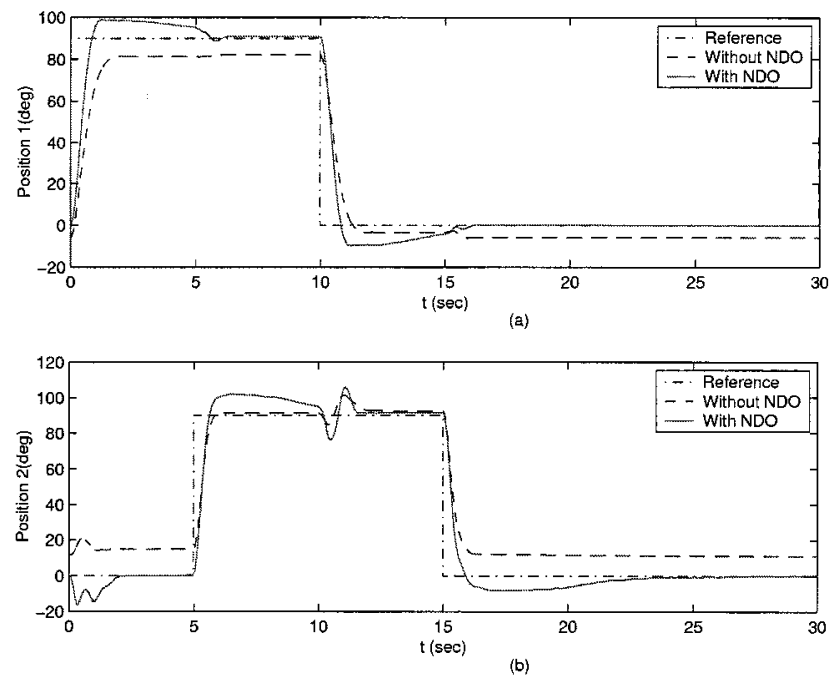

Fig. 8. Experimental results with and without DO: position.

Fig. 8 by a solid line. Compared with the tracking performance of the computed torque controller without the NDO, the performance is greatly improved using the friction estimation and compensation and the steady tracking error disappears. The outputs of the tachometers are also shown in Fig. 10 and the input voltages to the motors are shown in Fig. 11. It should be mentioned that, due to the measurement noise in tachometers, as in the computed torque controller, the velocity signals are filtered before being used in the NDO. 

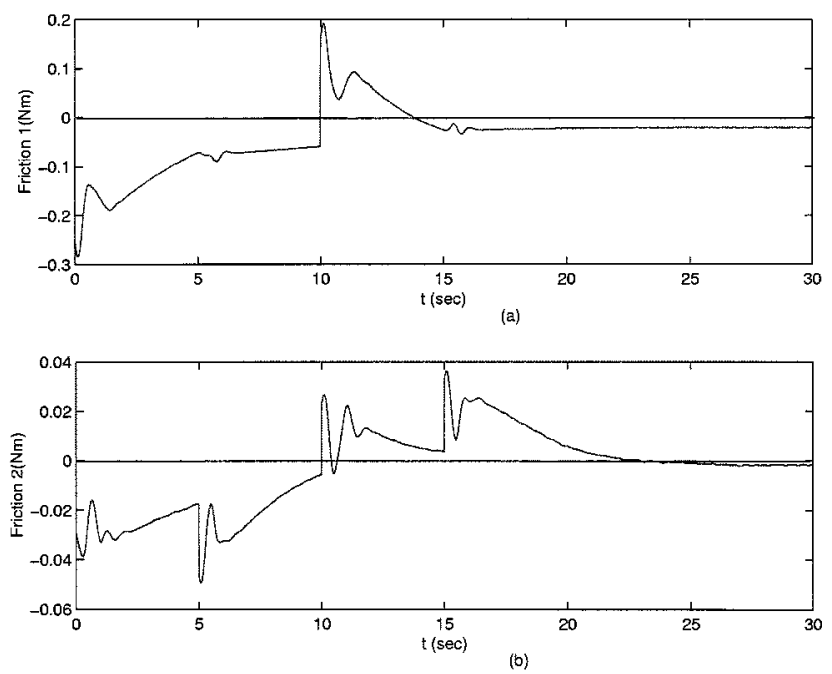

Fig. 9. Experimental results with DO: estimated friction.
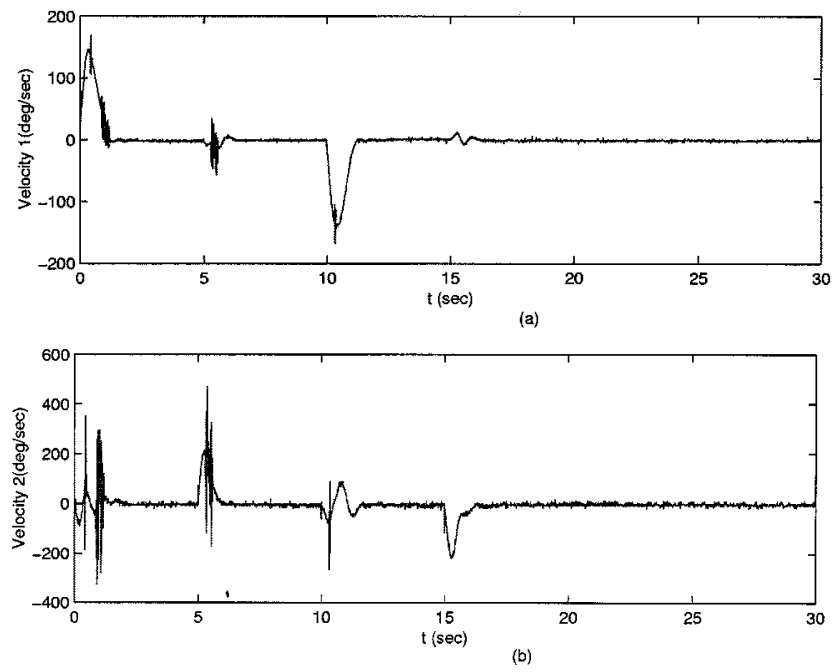

Fig. 10. Experimental results with DO: velocity.
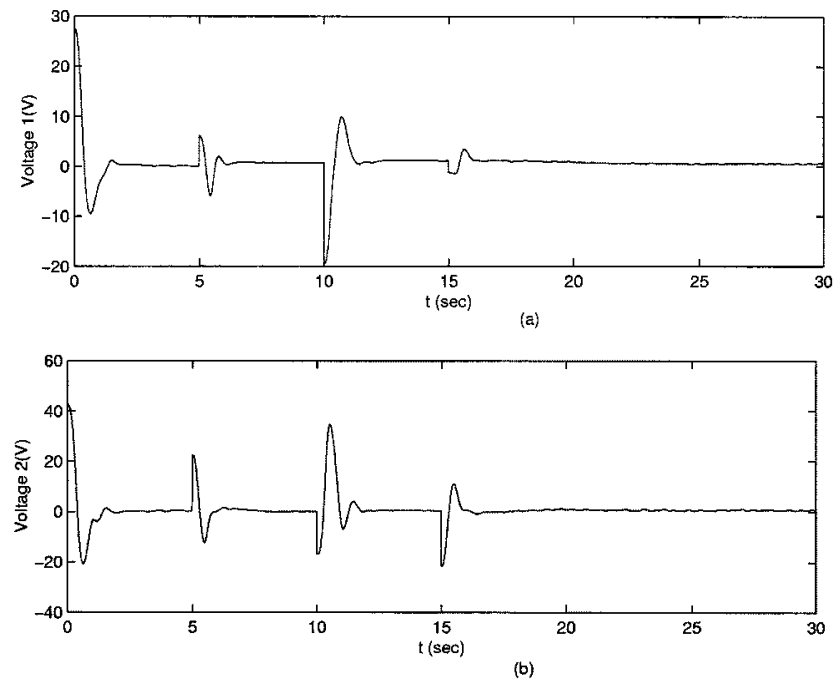

Fig. 11. Experimental results with DO: motor voltages.

\section{CONCLUSION}

This paper has presented a procedure for the design of an NDO for robotic manipulators. Following the procedure presented in this paper, a condition for convergence was established. The speed of the convergence can be specified by the design parameters. The proposed observer was tested by simulation and experiment. Even though the theory is developed for constant disturbances, it was shown that, for a rapid time-varying signal like friction, the observer exhibits satisfactory performance. By feedforward compensation of the estimated friction yielded by the DO, the performance, in particular, the steady-state performance, was significantly improved. The NDO proposed in this paper can also be applied in independent joint control, sensorless torque control, and fault diagnosis in robotics.

\section{ACKNOWLEDGMENT}

The authors greatly appreciate the comments and discussion by Dr. J. J. Gribble pertaining to the earlier version of this paper.

\section{REFERENCES}

[1] K. S. Eom, I. H. Suh, and W. K. Chung, "Disturbance observer based path tracking control of robot manipulator considering torque saturation," in Proc. Int. Advanced Robot. Conf., 1997, pp. 651-657.

[2] B. K. Kim, W. K. Chung, and Y. Youm, "Robust learning control for robot manipulator based on disturbance observers," in Proc. IEEE Ind. Electron. Conf., 1996, pp. 1276-1282.

[3] H. Olsson, K. J. Åström, C. C. de Wit, M. Gafvert, and P. Lischinsky, "Friction models and friction compensation," Eur. J. Control, vol. 4, no. 3, pp. 176-195, 1998.

[4] G. Zhang and J. Furusho, "Control of robot arms using joint torque sensors," IEEE Control Syst. Mag., vol. 18, no. 1, pp. 48-55, 1998.

[5] S. Komada and K. Ohnishi, "Force feedback control of robot manipulator by the acceleration tracing orientation method," IEEE Trans. Ind. Electron., vol. 37, pp. 6-12, Feb. 1990.

[6] S. Komada, K. Nomura, M. Ishida, and T. Hori, "Robust force control based on compensation for parameter variations of dynamic environment," IEEE Trans. Ind. Electron., vol. 40, pp. 89-95, Feb. 1993.

[7] T. Murakami, F. Yu, and K. Ohnishi, "Torque sensorless control in multidegree-of-freedom manipulator," IEEE Trans. Ind. Electron., vol. 40, pp. 259-265, Apr. 1993.

[8] S. P. Chan, "A disturbance observer for robot manipulators with application to electronic components assembly," IEEE Trans. Ind. Electron., vol. 42, pp. 487-493, Oct. 1995.

[9] K. Ohishi and H. Ohde, "Collision and force control for robot manipulator without force sensor," in Proc. IEEE Ind. Electron. Conf., 1994, pp. 766-771.

[10] H. N. Lin and Y. Kuroe, "Decoupling control of robot manipulators by using variable structure disturbance observer," in Proc. IEEE Ind. Electron. Conf., 1995, pp. 1266-1271.

[11] B. Friedl and Y. J. Park, "On adaptive friction compensation," IEEE Trans. Automat. Contr., vol. 37, pp. 1609-1612, Oct. 1992.

[12] S. Tafazoli, C. W. de Silva, and P. D. Lawrence, "Tracking control of an electrohydraulic manipulator in the presence of friction," IEEE Trans. Contr. Syst. Tech., vol. 6, pp. 401-411, May 1998.

[13] B. Bona and M. Indri, "Analysis and implementation of observers for robotic manipulators," in Proc. IEEE Int. Conf. Robot. Automat., Leuven, Belgium, 1998, pp. 3006-3011.

[14] P. J. Gawthrop and L. P. S. Smith, Metamodeling: Bond Graphs and Dynamic Systems. New York: Prentice-Hall, 1996.

[15] W.-H. Chen, D. J. Ballance, and P. J. Gawthrop, "Nonlinear generalized predictive control and optimal dynamic inversion control," in Proc. 14th IFAC World Congr, vol. E, Beijing, China, 1999, pp. 415-420.

[16] D. Karnopp, "Computer simulation of strick-stip friction in mechanical dynamic systems," ASME J. Dynam. Syst., Meas., Contr., vol. 107, pp. 100-103, 1985. 


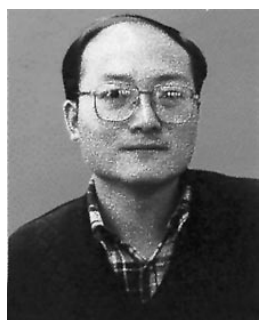

Wen-Hua Chen (M'00) received the M.Sc. and Ph.D. degrees from Northeast University, Shengyang, China, in 1989 and 1991, respectively.

From 1991 to 1997, he was a Lecturer in the Department of Automatic Control, Nanjing University of Aeronautics and Astronautics. He then held a research position in the Centre for Systems and Control, Department of Mechanical Engineering, University of Glasgow, Glasgow, U.K. He is currently a Lecturer at the Centre for Systems and Control, Department of Electronics and Electrical Engineering, University of Glasgow. His research interests include robust control, nonlinear control and their application in mechatronics, and aerospace engineering.

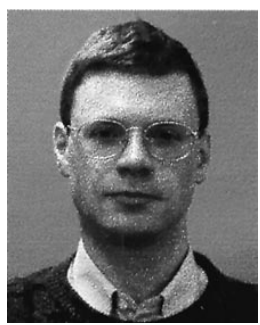

Donald J. Ballance (M'91) received the D.Phil. degree in control engineering from the University of Oxford, Oxford, U.K., in 1989.

Since then, he has been at the Centre for Systems and Control, Department of Mechanical Engineering, University of Glasgow, Glasgow, U.K., initially as a Research Assistant and currently as a Senior Lecturer. His research interests include modeling and analysis of systems using bond graphs, quantitative feedback theory and the effect of parameter uncertainty on control system performance, nonlinear control, and the practical application of these techniques.

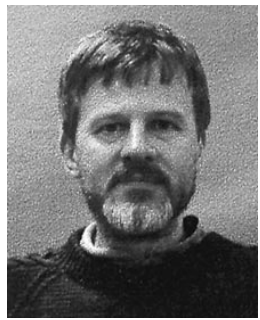

Peter J. Gawthrop (M'82-SM'94) was born in Seascale, Cumberland, U.K., in 1952. He received the B.A. (first class honors), D.Phil. and M.A. degrees in engineering science from Oxford University, Oxford, U.K., in 1973, 1977, and 1979, respectively.

Following a period as a Research Assistant with the Department of Engineering Science, Oxford University, he became a W. W. Spooner Research Fellow at the New College, Oxford. He then moved to the University of Sussex, initially as a Lecturer, and then as a Reader in control engineering. Since 1987, he has held the Wylie Chair of Control Engineering in the Department of Mechanical Engineering, Glasgow University, Glasgow, U.K. He was involved in founding the Centre for Systems and Control, which is a cross-departmental research grouping at Glasgow University. His research interests include self-tuning control, continuous-time system identification, and system modeling, particularly using bond graphs in the context of partially known systems. He is also interested in applying control techniques to a number of areas, including process control, robotics, aerospace systems, and anaesthesia. He has authored and co-authored approximately 120 conference and journal papers and three books in these areas. He was an associate editor of Automatica and an honorary editor of the Proceeding of the IEE, and serves on the editorial boards of a number of journals, including the IMechE Journal of Systems and Control, Journal of Process Control, IMA Journal of Mathematical Control and Information, the International Journal of Adaptive Control and Signal processing, and the European Journal of Control.

Dr. Gawthrop was the recipient of the 1994 Honeywell International Medal presented by the Institute of Measurement and Control

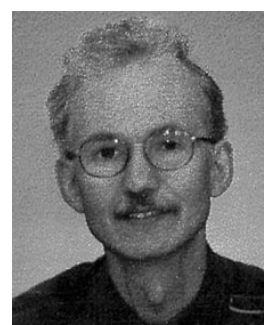

John O'Reilly (M'82-SM'00) received the B.Sc., $\mathrm{Ph} . \mathrm{D}$., and D.Sc. degrees in engineering from Queen's University, Belfast, Belfast, Northern Ireland, in 1972, 1976, and 1985, respectively.

$\mathrm{He}$ is currently a Professor of control engineering at the Centre for Systems and Control, Department of Electronics and Electrical Engineering, University of Glasgow, Glasgow, U.K. He is currently a Visiting Erskine Fellow in the Department of Electrical and Electronic Engineering, University of Canterbury, Canterbury, New Zealand. His main research interests are centered on integrated and nonlinear control, with particular application to aerospace and deregulated power systems. He is also a Consultant Editor for the International Journal of Control.

Dr. O'Reilly was the recipient of a U.K. Royal Academy of Engineering Foresight Award. 